\title{
Exercise Related Sudden Death: The Changes of Expression of Bax, Bcl-2 Protain in Myocardial Tissue and Brain Motor Cortex
}

\author{
Yu QIAN ${ }^{1, a}$, Ren-Jun YANG ${ }^{1}$ and Jin YIN $^{1, b, *}$
}

${ }^{1}$ ChengDu Sport University Institute of Sports Medicine and Health, ChengDu Sport University, WuHou District, ChengDu, SiChuan province, Postal Code 610041

a676609747@qq.com

b791083068@qq.com

*Corresponding author

Keywords: Exercise related, Sudden, Death-Exercise-Fatigue-Bax -BCl-2-LDH-2-BDNF.

\begin{abstract}
Purposes: Study the changes of cardiac and brain function of exercise related sudden death in rats under over fatigue state and explore the causes of sudden death preliminarily. Methods: Totally 72 male SD rats.7 rats were randomly selected as negative control group. The others were trained continuously with exhaustive loaded-swimming exercises to achieve the over fatigue state. Collect suffering-sudden death rats during and after exercise within 24 hours. Results: 1.Myocardial fibers were attenuated and some of fibers ruptured.2.Neuron is disordered in motor cortex slice, cells with a looser stroma.3.The level of serum LDH-2 sudden death group was slightly higher than negative control group.4.The expression level of Bax protein were increased in fatigue and sudden death group $(\mathrm{P}<0.05)$, while Bcl-2 protein decreased in heart tissue but increased in brain motor cortex $(\mathrm{P}<0.01)$. Conclusions: The changs of expression of Bax, Bcl-2 and BDNF maybe related to the protection-inhibition mechanism. Changes of Bax and Bcl-2 protein may be one of the causes of movement induced sudden death.
\end{abstract}

\section{Introduction:}

Exercise related sudden death is a serious practical problem in the field of sports medicine that has long been widely concerned. Compared with other sport disorders, exercise related sudden death rate is not common, but directly life-threatening[1].It is reported that $70.73 \%$ of all exercise related sudden deaths are sudden cardiac deaths, and $18.27 \%$ are the brain death[2].According to resources from World Health Organization, International Society of Cardiology and American Heart Association, we defined exercise related sudden death as follows: the accidental death of symptomatic or asymptomatic athletes and individuals in physical activities during or after exercise within 24hours[3].Motor cortex is the nerve center of controlling the body motion. If its morphologiy, structure or function changes, the ability of the body's movement and other functional state will be affected, including the heart. Movement alone could not induce sudden death, unless collaborating with underlying heart diseases, but both one-time strenuous exercise and intensive training for a long time will damage cerebral motor cortex..Admittedly, sports training have specific effects on cardiac muscles. Adaptive compensation would take place after a period of exercise, such as appear enlarged heart, cardiac hypertrophy, increasing numbers of mitochondria, thickened myofibrils and improved cardiac reserves[4].However, in the process of exercise induced over fatigue, what happens about cardiac construction and 
functional and the construction and function of damaged motor cortex is rarely reported. Many studies point out that excessive exercise training could cause irreversible pathological damages in heart and brain motor. During the process of excessive exercise, myocardial oxygen demand increases, together with ascending level of catecholamine and sympathetic nervous activity, which causes vasospasm and myocardial ischemia, triggering fatal arrhythmia or myocardial infarction, ending with sudden death. When damage to stimulate the brain, motor cortex will secrete a protective factor BDN(brain derived neurophic factor, the brain-derived neurotrophic factor, To maintain their normal morphology and protect their normal physiological function, over fatigue caused the cells of motor cortex increased, but mechanism of body protection to control cells death[5].According to these theories, we take 36 hours excessive recovery fatigue model on the basis of glycolysis energy supplying: to train rats continuously with exhaustive loaded-swimming exercises to achieve the over fatigue state. We collected the rats of sudden death and observed related pathological and physiological indicators, such as myocardial tissue and brain motor and serum LDH-2, Bax, Bcl-2 and BDNF. By studying changes of above indexes associated with exercise related sudden death, we try to provide a further understanding and better judgement for exercise related sudden death, thus preventing its happening.

\section{Materials and Methods}

\section{Materials}

A total of 72 purebred male SD rats, SPF grade, 3 months old, were purchased from Chengdu Da-Shuo Biological science and Technology ltd. Animals were divided 6 per cage in the animal-room under natural lighting, with temperature setting at $20 \sim 28{ }^{\circ} \mathrm{C}$ and relative humidity of $55 \%$ to $65 \%$. Free feeding with standard rodent chow and drinking water were provided. The animal room maintained quiet and ventilative. The cages were cleaned and drinks and food were changed every morning.

\section{Grouping}

7 rats were randomly selected as the blank control group $(\mathrm{N})$, others were trained continuously with exhaustive loaded-swimming exercises to achieve the over fatigue state. The end of each training is marked by the immediate sank when rats had been put into the water again after last rest. After reaching negative superposition state of excess recovery after the third 36-hours-training cycle under fatigue model, seven rats were randomly sacrificed as fatigue group (group C).After reaching negative superposition state of excess recovery under fatigue model, rats died during or after each training within 24 hours were collected as exercise-related sudden death group (group D), excluding rats died of suffocation.(exclude the rats that died due to choking water).

\section{The Animal Model}

The experimental group was given adaptive swimming pre-training for one week in the $50 \times 50 \times 100 \mathrm{~cm}$ transparent glass water vat. The water temperature was controlled at $31{ }^{\circ} \mathrm{C} \pm 2{ }^{\circ} \mathrm{C}$ with room temperature of $21{ }^{\circ} \mathrm{C} \sim 23{ }^{\circ} \mathrm{C}$ and relative humidity of $40 \%-60 \%$. The depth of water is 1.5 times $\times$ (body length + tail length). We gradually increased the load to $12 \%$ of rat's body weight and extended the time of nonstop swimming over 2 min.During the second week, according to the fatigue model based on glycolysis of Yin-Jin ${ }^{[5]}$, we established the over fatigue animal model as follows. Under the Load for $12 \%$ of rats body weight, rats to swimming exercise 
according to large middle and small amount of exercise (large $\mathrm{N}=4$ times, middle $\mathrm{N}=3$ times, small $\mathrm{N}=2$ times).Every morning and afternoon complete two groups exercise, 5 min rest after every times, and 15 min rest after every group ${ }^{[6]}$.Every swimming time is according to rat nose sank underwater to prevail. After modeling, rat according to a maximal training to swimming per $36 \mathrm{~h}(2$ groups $\mathrm{X} 4$ times, load $12 \%$ of body weight),After the seventh $36 \mathrm{~h}$, the rats sports ability recovery showed negative superposition state, recovery capability decreased, fatigue continuously accumulation ${ }^{[7]}$.After the seventh $36 \mathrm{~h}$ swimming cycle, laboratory technician take turns on duty in the laboratory, let rats in the water vat to swim, when rats sink to under the water surface again after the first rest then to end the experiment; rats dead in exercise or after exercise that within 24h,drawing materials immediatly as exercise-related sudden death group(D group)(Excluding deaths rat that due to choking water); Throughout the experiment appeared five rats that cases of movement-related sudden death. The amount of exercise and the sports arrangements such as table 1 .

Table 1 Rats swimming training arrangements

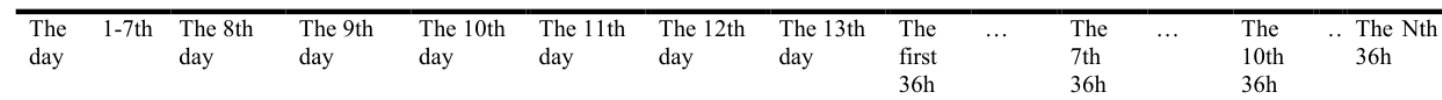

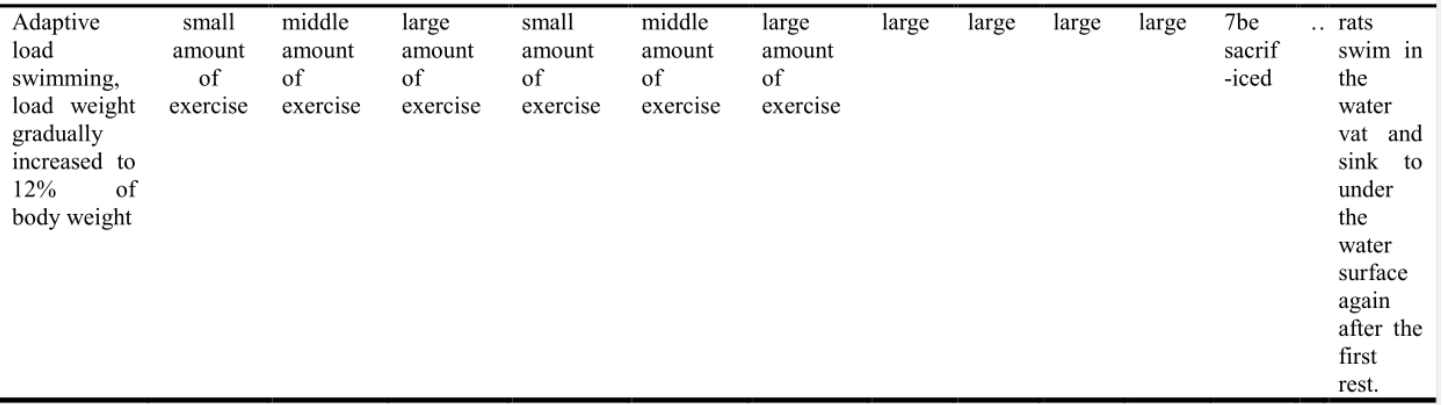

\section{Specimen Collection}

Death in rat immediately take the femoral artery mixed blood 5 8ml, after standing $30 \mathrm{~min}, 3000 \mathrm{rpm} / \mathrm{min}$ centrifugal $15 \mathrm{~min}$, The supernatant was used for LDH-2 detected. After that, immediately cut off its head and opens its skull to take out complete brain and fixed in 10\% neutral Formaldehyde Solution, then supine position and along the sternum midline longitudinal incision of about $10 \mathrm{~cm}$, complete removal of the rat heart, after washing by cold saline, filter paper blots, fixed in $10 \%$ neutral Formaldehyde Solution, for the production of sliced. Blank control group of rats use $0.3 \%$ sodium pentobarbital solution $40 \mathrm{mg} / \mathrm{Kg}$ intraperitoneal injection of anesthesia, using the same method to prepare blood serum, then complete heart and brain rapidly removed into $10 \%$ neutral formaldehyde solution, fixed $24 \mathrm{~h}$,replacing a solution after fixation $2 \mathrm{~h}$. 


\section{The Test of Slice and Sera:}

HE Staining and Immunohisto Chemistry. Take out heart tissue and brain that fixed in $10 \%$ neutral formaldehyde solution, use water to rinsing residual fixative; after resection of the apical part of left ventricle of the heart and the cerebral cortex motor area, turn into the $70 \%, 80 \%, 90 \%, 95 \%$, anhydrous alcohol to dehydrated; after dehydration, with xylene transparent, baptist wax, embedded in paraffin, put wax block in $4{ }^{\circ} \mathrm{C}$ refrigerator until ready to use. Remove paraffin blocks from the refrigerator. Along the frontal plane to continuous to take $4 \mu \mathrm{m}$ sections. After dewaxing with xylene,then successively put it into 100\% ethanol,95\% ethanol,85\% ethanol,70\% ethanol,50\% ethanol until distilled water, dry it with absorbent paper then start of hematoxylin - eosin staining, and watching myocardial cell fibers and Motor cortex neurons, and the changes of morphology and structure under an optical microscope after mounting.

The remaining sections using immunehisto chemical expression of SP (streptavidin-peroxidase) staining marks of Bax and Bcl-2 and SABC (streptavidin biotin-peroxidase complex) staining marks of BDNF. Used a rat anti-Bax, Bcl-2 and BDNF monoclonal antibody concentrate $0.2 \mathrm{ml}$ (borrow from an American company which named abcam), The three sections of Bax, Bcl-2 and BDNF in turn after dewaxing to water, rinsing by PBS, incubating at room temperature with $3 \%$ $\mathrm{H} 2 \mathrm{O} 2$, rinsing by PBS,5\% normal goat serum closed, adding dropwise the first antibody to incubated with $24 \mathrm{~h}$ under $4{ }^{\circ} \mathrm{C}$, adding dropwise the second antibody to incubated with $10 \mathrm{~min}$ under $37^{\circ} \mathrm{C}$; adding dropwise immunohistochemistry peroxidase solution was incubated, rinsing by PBS, the DAB colors, rinsing by tap water, hematoxylin staining,graded alcohol dehydration, xylene transparent, gummi mounted. Observing by light microscopy after the completion of the immunohisto chemical staining,collecting chart, using Image pro-Plus image analysis software Bax, Bcl-2 protein and BDNF expression in Myocardial tissue and Brain motor cortex.

Serum Enzyme-linked Immunoassay for Detection of LDH-2. This LDH-2 ELISA kit is intended Laboratory for Research use only and is not for use in diagnostic or therapeutic procedures. The Stop Solution changes the color from blue to yellow and the intensity of the color is measured at $450 \mathrm{~nm}$ using a spectrophotometer. The calibration standards are assayed at the same time as the samples and allow the operator to produce a standard curve of Optical Density versus LDH-2 concentration. The concentration of LDH-2 in the samples is then determined by comparing the O.D.of the samples to the standard curve.

\section{Statistical Methods}

All raw data are input SPSS11.5 statistical software for analysis and processing.Each set of sample data measured as mean \pm standard deviation $(\mathrm{X} \pm \mathrm{SD})$ representation, between groups using independent sample T-test.significance level $\alpha=$ 0.05 fetch, $\mathrm{P}<0.05$ was significant difference, $\mathrm{P}<0.01$ was a very significant difference.

Biochemical indicators were respectively detection in affiliated Hospital Laboratory of Chengdu Sports University and the Experimental Medicine and Biochemical detection chamber of West China Hospital of Sichuan University. 


\section{The Results}

\section{The Results of HE Staining of Myocardial Tissue and the Cortical Motor Neuron}

The results of $\mathrm{HE}$ staining showed that, group $\mathrm{N}$ rat cardiomyocytes fibers were arranged in neat rows, uniform dense stromal cells, no abnormalities.Group C myocardial fibers visible slim, partial rupture occurs. Myocardial blood vessels of sudden death Group D is extreme expansion, most of myocardial fiber breakage, cardiac congestion and appeared severe hemorrhage and necrosis, myocardial fibers dissolve necrotic(Figure 1)

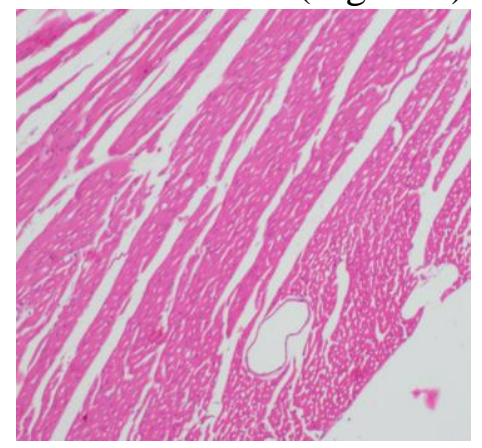

$\mathrm{N}$

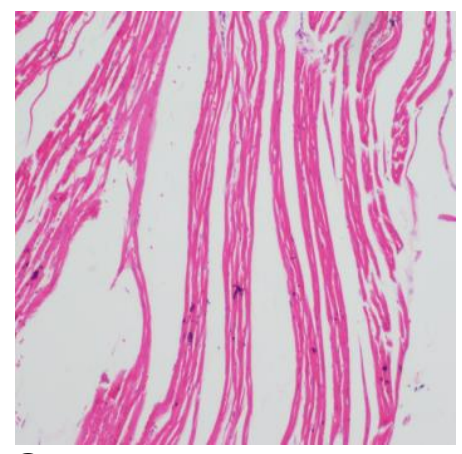

C

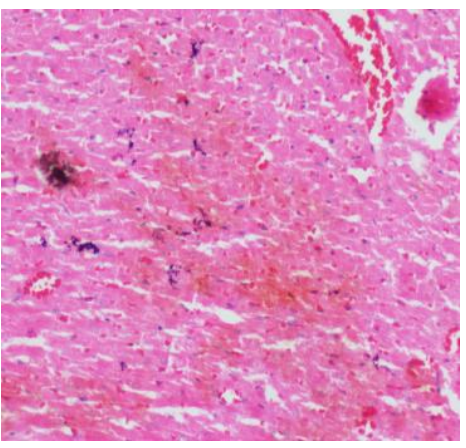

D

Figure 1.Blank group(N),Fatigue group(C)Sudden death group(D)

Comparison of myocardial cell morphology(200X)

$\mathrm{N}$ group cells of motor cortex arranged well, intercellular substance is close ,no pathological change; $\mathrm{C}$ group neuron is disordered ,intercellular substance is loose, parts of never fiber are entangled, but cell structure is well ; D group cells of motor cortex is disordered, intercellular substance is close even bubbled, microglias cells, meninges and blood vessels in brain expanded extremely, neuron fibers are entangled, neuron caused red generation, parts of neuron show focal necrosis, forming vascular cuffing.(Figure 2).

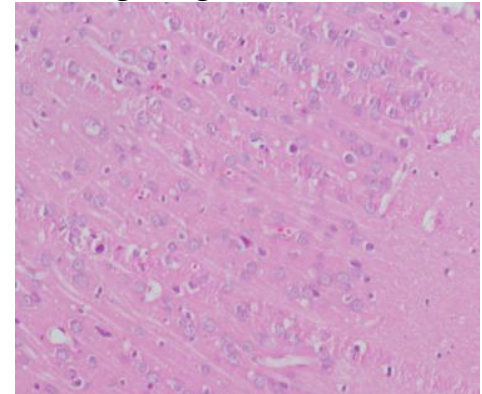

$\mathrm{N}$

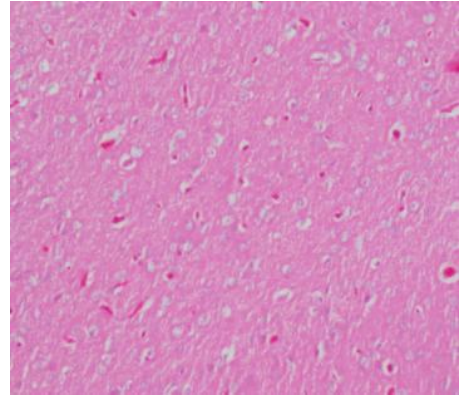

C

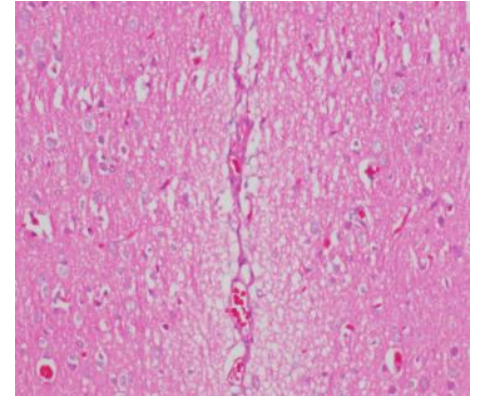

$\mathrm{D}$

Figure 2.Blank group (N),Fatigue group(C)Sudden death group(D)

Comparison of motor cortex cell morphology (200X)

\section{Serum LDH-2 Test Results}

Serum test results showde that, the content of group N serum LDH-2 was lower, the content of fatigue group $\mathrm{C}$ and Sudden death group D serum LDH-2 were higher (Figure 3).At the same time, group $\mathrm{C}$ was significantly higher than the group $\mathrm{N}(\mathrm{P}<0.05)$ (Table 2), the content of group D serum LDH-2 was lower than group C. 
Table 2. Serum test results showed that the differences between-group content of LDH-2.

\begin{tabular}{lll}
\hline group & Sample size(n) & LDH-2 \\
\hline $\mathrm{N}$ & 7 & $7.16 \pm 0.122$ \\
\hline $\mathrm{C}$ & 7 & $7.92 \pm 0.304$ \\
\hline $\mathrm{D}$ & 5 & $7.47 \pm 0.233^{*}$ \\
\hline
\end{tabular}

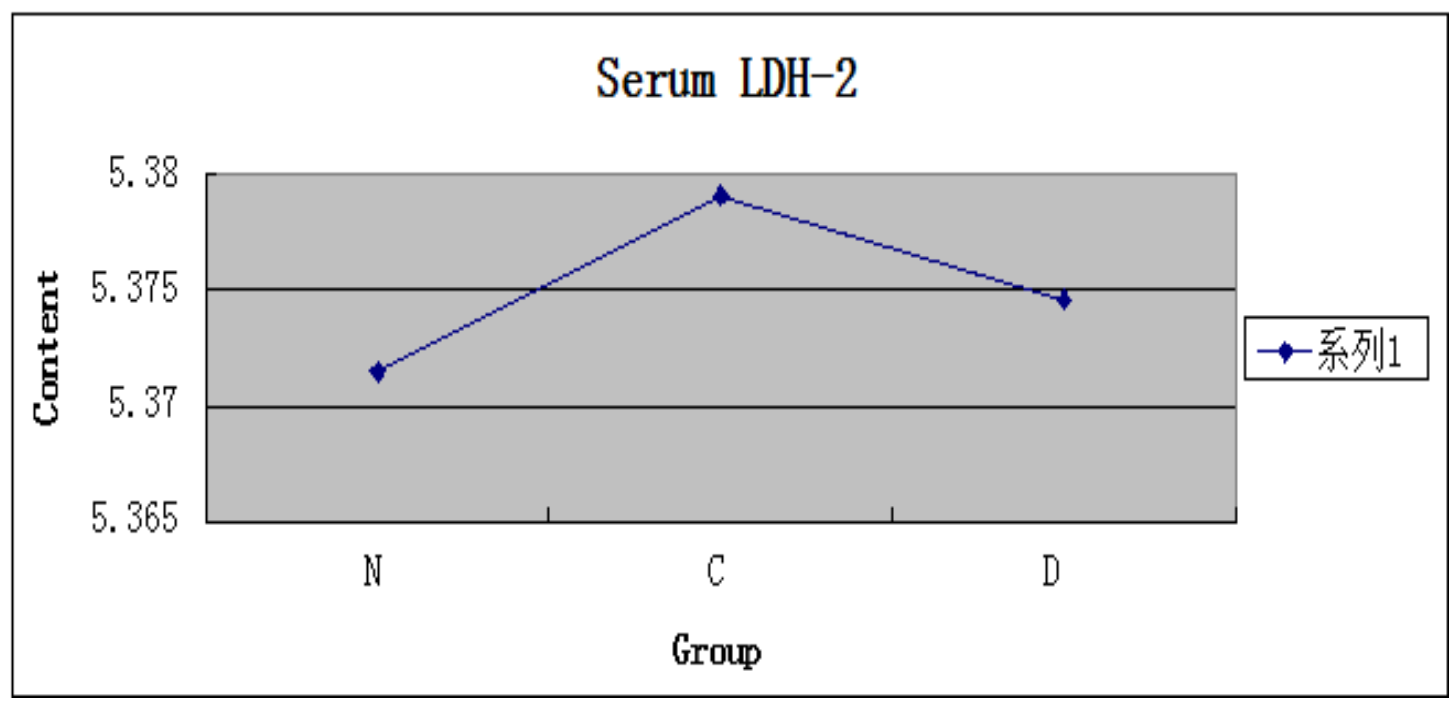

Figure 3.group N, group C,group D serum LDH-2 content trends

\section{Immunohisto Chemical Detection of Bax, Bcl-2 Expression Levels in Myocardial Tissue and Brain Motor Cortex}

Bax Promote Apoptosis Gene Expression Changes. Figure 4,5 shows Bax expression by $\mathrm{N}$ group was weaker than in group $\mathrm{C}$ and group $\mathrm{D}$, as shown in Table 3 , the results after the SPSS statistical software processing, compared with the blank control group, the Bax protein levels in rat heart tissue and brain motor cortex of sudden death group D were significantly increased, it has a very significant statistical significance $(\mathrm{P}<0.01)$, rat heart tissue fatigue group $\mathrm{C}$, the Bax protein content increased, it has a statistically significant $(\mathrm{P}<0.05)$. The expression between the three groups showed an increasing relationship .

Table3.Bax protein expression

\begin{tabular}{cccc}
\hline group & Sample size $(\mathrm{n})$ & In heart tissue & In brain motor cortex \\
\hline $\mathrm{N}$ & 7 & $1.58 \pm 0.43$ & $0.81 \pm 0.72$ \\
\hline $\mathrm{C}$ & 7 & $1.91 \pm 0.13 *$ & $2.79 \pm 1.23^{*}$ \\
\hline $\mathrm{D}$ & 5 & $2.36 \pm 0.03 * *$ & $3.52 \pm 1.76^{* *}$ \\
\hline
\end{tabular}

Note: compared with the blank control group, $* \mathrm{P}<0.05 \quad * * \mathrm{P}<0.01$. 


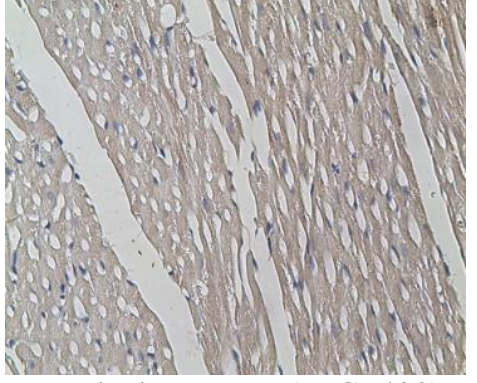

Blank group N $(\mathrm{IHC} \times 400)$
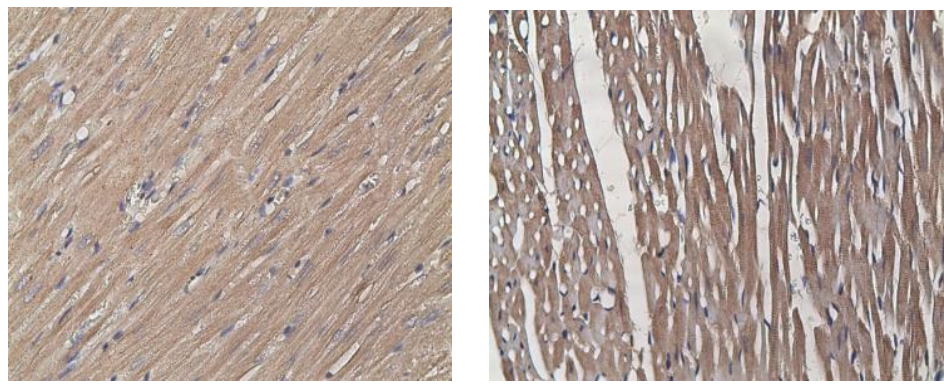

Fatigue grou C ( $\mathrm{HHC} \times 400) \quad$ Sudden death group D $(\mathrm{IHC} \times 400)$

Figure4.group N,group C,group D,the figure of heart tissue's bax protein expression in the mean.
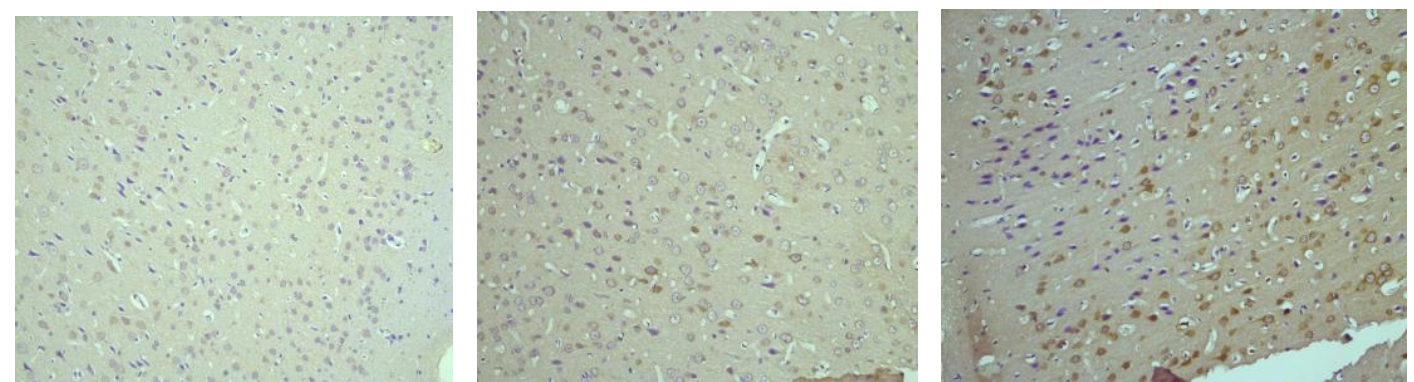

Blank group N $(\mathrm{IHC} \times 400) \quad$ Fatigue grou C $(\mathrm{IHC} \times 400) \quad$ Sudden death group D $(\mathrm{IHC} \times 400)$

Figure5.group N,group C,group D,the figure of brain motor cortex's bax protein expression in the mean.

Bcl-2 Inhibits Apoptosis Gene Expression Changes. The statistics results from Table 4 showed that,compared with the blank control group,the rat heart tissue of Fatigue group $\mathrm{C}$ and sudden death group $\mathrm{D}$, the Bcl-2 protein were significantly lower, Showing a increasing trend(Figure 6), it has a very significant statistical significance $(\mathrm{P}<0.01)$. But the expressions of $\mathrm{Bcl}-2$ protein are higher in brain motor cortex of $\mathrm{C}$ and $\mathrm{D}$ group,Figure 7 shows the positive expression of $\mathrm{C}$ and $\mathrm{D}$ group $\mathrm{Bcl}-2$ than in group $\mathrm{N}$ were higher,Showing a decreasing trend.

Table4.Bcl-2 protein expression

\begin{tabular}{cccc}
\hline group & Sample size $(\mathrm{n})$ & In heart tissue & In brain motor cortex \\
\hline $\mathrm{N}$ & 7 & $2.30 \pm 0.27$ & $1.99 \pm 0.76$ \\
\hline $\mathrm{C}$ & 7 & $1.79 \pm 0.12 * *$ & $7.11 \pm 1.89 * *$ \\
\hline $\mathrm{D}$ & 5 & $1.29 \pm 0.32 * *$ & $8.65 \pm 3.70 * *$
\end{tabular}

Note:compared with the blank control group, $* \mathrm{P}<0.05 \quad * * \mathrm{P}<0.01$
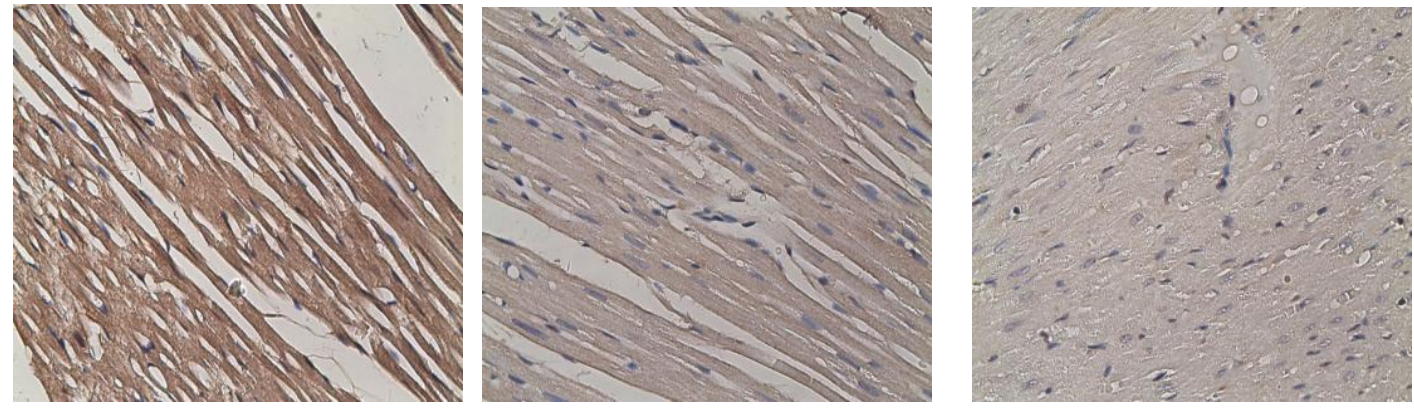

Blank group $(\mathrm{N})(\mathrm{IHC} \times 400) \quad$ Fatigue group $(\mathrm{C})(\mathrm{IHC} \times 400) \quad$ Sudden death group $(\mathrm{D})(\mathrm{IHC} \times 400)$

Figure6.Immunohistochemical staining the expression of Bcl-2 in myocardial tissue of rats(400X) 

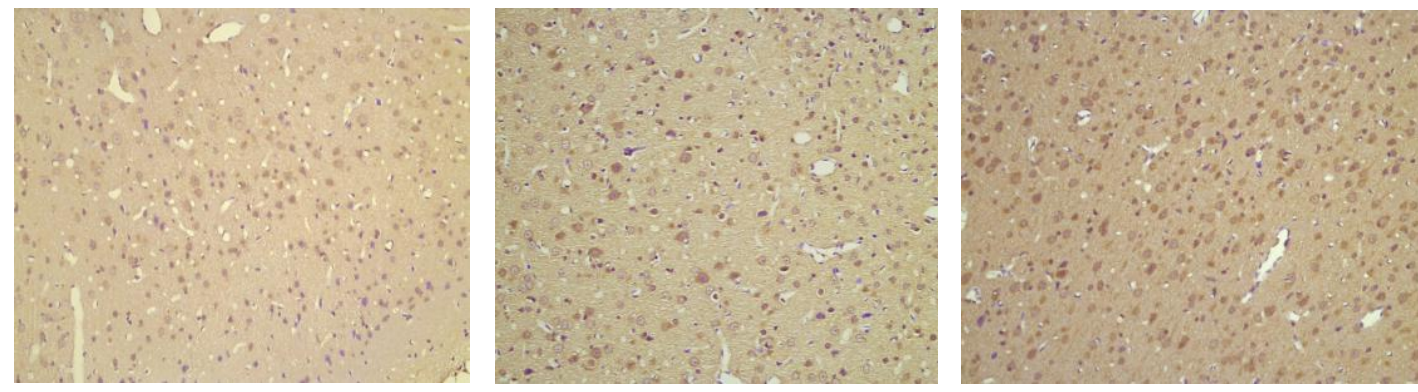

Blank group $(\mathrm{N})(\mathrm{IHC} \times 400) \quad$ Fatigue group $(\mathrm{C})(\mathrm{IHC} \times 400) \quad$ Sudden death group $(\mathrm{D})(\mathrm{IHC} \times 400)$

Figure7.Immunohistochemical staining the expression of Bcl-2 in brain motor cortex of rats(400X)

\section{Immunohisto Chemical Detection of BDNF Expression Levels in Brain Motor Cortex}

$\mathrm{N}$ group BDNF express minimal, but D group BDNF express higher than $\mathrm{N}$ group,but lower than $\mathrm{C}$ group is the results showed $(\mathrm{P}<0.01)$. (Figure 8, Table 5)

Table5.BDNF expression

\begin{tabular}{ccc}
\hline group & Sample size $(\mathrm{n})$ & $\mathrm{BDNF}$ \\
\hline $\mathrm{N}$ & 7 & $0.25 \pm 0.13$ \\
\hline $\mathrm{C}$ & 7 & $3.31 \pm 1.83^{* *}$ \\
\hline $\mathrm{D}$ & 5 & $2.20 \pm 1.35^{* *}$ \\
\hline
\end{tabular}

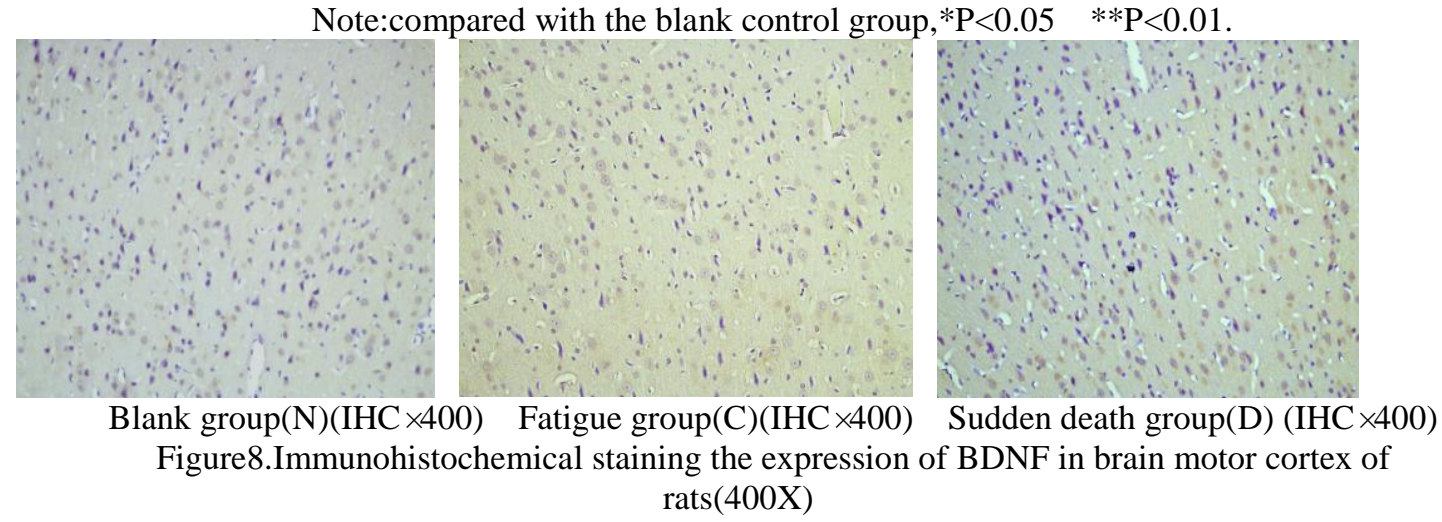

\section{Analysis and Discussion}

\section{Changes of Morphology and Cell Structure of Myocardial Fiber and Cortical Motor Neuron}

Exercise-related sudden death usually betide persons that has long been engaged in sports and 'health' in peacetime, Its sudden brought great difficulties for prevention work, as such, for those engaged in strenuous exercise people early detection of morphology structural abnormalities of cardiac cell and cerebral cortical neuron is very important. Sustained heavy load games make myocardial cells and cerebral cortical neuron showed irreversible pathological changes, Long-term overload movement, even athletes mental status and muscle also keep the excited state so that the body movement is at a high level, but the potential cardiac and brain neure pathologic changes may cause exercise-related sudden death occurred[8].

Myocardial large strength of systolic in a state of Long-term fatigue, lack of completely recovery is a direct factor that leading to myocardial damage. Results can be seen by HE staining, blank group myocardial cells fibers arranged closely uniform. 
The reason why fatigue group cardio myocytes fibers tenuity and blood vessels utmost dilate ,may be due to in the long-term process of a large load overtraining, muscle filaments contractile protein long time activity, ATP lack or calcium and magnesium ions aggregation and changes, inadequate energy supply, myocardial fibers extreme fatigue have not been the timely and fully recovery[9].Sudden death group myocardial blood vessels extreme expansion, most of the muscle fibers appear broken, myocardial hyperemia severe bleeding severe necrosis, necrotic myocardial fibers dissolve. Because when strenuous exercise, the body is in stress, increase oxygen consumption, increased cardiac do work and appears hypertonic dehydration during exercise, blood in highly viscous state, prone to coronary artery spasm and spasm lifted reperfusion coronary endothelial injury and thus lead to damage and regional ischemia-induced platelet aggregation, thrombosis, leading to myocardial ischemia, ischemia, energy supply inadequate to appeared metabolic disorders[10].The main cause of exercise-related sudden cardiac death is myocardial ischemia and, myocardial infarction, secondary cardiac hypertrophy induced relative myocardial ischemia, myocardial metabolism disorders[7]. Rise in blood pressure during exercise, during exercise or movement suddenly stops blood pressure dropped to unusually low levels; in addition, with breath holding related Valsalva effect causes chest negative pressure decreased significantly impede left ventricular filling, so that cardiac output is greatly reduced, will increased myocardial ischemia[11].

Long-term scientific training can have a positive impact on the morphology and function of the heart, such as cardiac volume increases, wall thickening, ventricular enlargement, stroke volume increased, resting heart rate slowed, etc. Such a big heart and a slow heart rate is called "athlete heart". that are athletes adapt to training stimulus results, its have a great role in promoting to improve athletic performance. But inappropriate exercise training intensity and too much exercise caused overtraining is easy to cause exercise-related myocardial injury [12].

For a long period of time strenuous exercise can caused the oxygen in the body are in short supply, also imbalance between oxygen supply and oxygen consumption, the anoxia is called motility of oxygen, It is a relative state of lack of oxygen. Motility hypoxia influenced the brain tissue of aerobic metabolism to maintain normal physiological function. During strenuous exercise, motor cortex in a relatively state of chemia oxygen deficit, the quantity of oxygen free radicals (OFR) in neurons will increased, neurons was injuried because of calcium is overload[13].

In over fatigue state, body will produced large quantity of oxygen free radical, disorder of internal environment the decline of physique. The body of the continuous overlay caused by oxygen free radicals in the motor cortex to produce too much more than the body's own ability to make clear and can't be clear in time, because of fatigue recovery ability declining, successive periods of 36 hours weight loading swimming movement makes the motor cortex of rats in cannot be cleaned in time, free radicals caused by oxygen free radicals accumulation, cause a series of changes in the motor cortex cell morphological structure. the experimental model of rat weight to swimming, for a long period of time in intense exercise makes the rats had motility anoxic condition[14]. When the motor cortex is in a state of hypoxia, under the stimulus of oxygen will pass its regulating mechanism to maintain its normal morphology and function. It Can be seen from the HE staining, the motor cortex neurons arranged disorder, interstitial cells loose appear even cavity, in the motor cortex capillary expansion, part of the nerve fiber winding, some neurons occurred red degeneration, shows that continuous great physiological load of exercise fatigue state weight loading swimming to the motor cortex blood anoxic injury stimulation, and in 
the condition of fatigue with the overlay, the damage of fatigue stimulate the enhance the motor cortex injury.

\section{Serum LDH-2 Changes and its Impact}

Lactate dehydro genase 2 (LDH-2) is a glycolytic enzyme.Lactate dehydro genase can catalytic pyruvate to lactic acid, it exists in almost all tissues. By detecting This indicator can observed and diagnosis myocardial disease, when myocardial damaged ,this enzyme tend to have elevated.LDH catalytic reduction and oxidation reaction between pyruvate and lactate, under alkaline conditions can promotes the reaction of lactate to pyruvate direction, whereas under neutral conditions can promote the conversion of pyruvate to lactate(called as the reverse reaction).LDH is a key enzyme involved in sugar anaerobic glycolysis. Cardiomyocytes LDH activity is much higher than serum, which the content of LDH-1 and LDH-2 is highest, and LDH-2 occupied a dominant position. When acute myocardial infarction, serum LDH-2 significantly increased.LDH-2 is usually used as an effective tool for the diagnosis of myocardial infarction. The study found that rats in the state of fatigue, $\mathrm{LDH}-2$ were significantly increased, in the process of rats in prolonged heavy load training that lead to over fatigue ,myocardial infarction may occur, resulting in movement-related sudden cardiac death[15].

\section{Myocardial Cell and the Expression Change of Bax. BCL-2 Protein}

When exhaustive exercises result in the body fatigue, due to overload, exceeding the physical adaptations, this will cause organ damage. Cardiac injury is one of the most common organ exhaustion damage, it characterized by myocardial apoptosis genes, form and structure of myocardium, cardiac biochemical, cardiac electrophysiology etc. When fatigue, heart damage can lead to abnormal myocardial enzyme, arrhythmia, cardiac function reduced, cause exercise sudden death [16]. Excessive myocardial cell apoptosis is one of the ways of myocardial cell damage, myocardial cell damage caused by stress related to cell excessive apoptosis [17]. Studies have found that early emergence of exercise-induced cardiac hypertrophy myocardial cell apoptosis, then apoptosis phenomenon disappeared but cardiac hypertrophy appeared. Therefore, you could suggest that myocardial hypertrophy caused by the exercise spurs may be related to myocardial cell apoptosis firstly and then enter the cell proliferation. Arrhythmia and sudden completely block can lead to sudden death, James thought myocardial cell apoptosis participated in the reconstruction process of the shape of sinoatrial node, atrioventricular node and His buddle, Block ectopic residue or damage caused by incomplete reconstruction or excessive reconstruction is an important cause of cardiac arrhythmias and conduction block [18].

Bax, BCL-2 Protein, Respectively have the function of inhibition and promote cell apoptosis,they play an important role in regulating in cell apoptosis. By stabilizing mitochondrial membrane,BCL-2 Protein which distributed in outer membrane of mitochondria, preventing promote apoptosis related proteins in the mitochondria leak into cytoplasm and blocking the $\mathrm{Ca}^{2+}$ release from the endoplasmic reticulum, blocking apoptosis by reducing the endonuclease activity,the endonuclease is rely on $\mathrm{Ca}^{2+}$. Bax is protein relates to the BCL-2 protein, it can take the shape of dimer form different sources with antiapoptotic proteins $B C L-2$, inhibit the function of the BCL-2, which can promote cell apoptosis.Therefore,Bax is one of the important controlling gene cell apoptosis.

The results of the study showed that sudden death myocardial Bax protein expression increased significantly than the blank group, while the BCL-2 protein 
expression significantly reduced. It showed that in the condition of fatigue, rat myocardial cell apoptosis rate was accelerated, cell apoptosis in myocardial infarction, then cell fibers necrosis happened in the infarction area. Apoptosis can damage or clean necrosis cells, plays certain positive role in keeping the cells in a steady state [19].

But the myocardium is made of terminal differentiation cells, they do not have proliferative capacity, their apoptosis inevitably lead to the reducing of myocardial cells. During the movement, the heart cavity blood filling increases, cardiac output increases, and tension on the myocardial cells aggravated, myocardial tissue has ischemia and hypoxia relatively than it at ordinary times, which cause cell apoptosis. Long-term excessive exercises lead to the increasing of myocardial cell apoptosis; it may be one of the mechanisms of early phase exercise-induced myocardial damage.

In this experiment, blank control group, fatigue group, sudden death group, if the tendency that their Bax increased and BCI-2 decreased has reaction at the tendency that myocardial cell apoptosis and inhibit apoptosis factors in the metabolism of physiological balance was destroyed. When the balance of Bax and BCL-2 was damaged to a certain point (limit), it damaged myocardial tissue, which leading to the cardiac exercise-induced sudden death.

\section{The Expression Change of Bax.BCL-2 Protein in Brain Motor Cortex}

The expression of Motor cortex of Bax protein in cells and the Bcl-2 protein control the regulation of cell apoptosis, in the condition of excessive fatigue, continuous intensive weight loading swimming under a long period of chemia hypoxia injury, caused increasing of the motor cortex cells apoptosis, the regulation mechanisms of cell apoptosis in the motor cortex is activated, the expression of genes related to cell apoptosis regulation becomes active.When blood anoxic injury stimulation, in the motor cortex increased Bax gene expression, Bax protein synthesis increase [20].Studies have found that tissue oxygen free radicals in cells showed positive correlation between the quantity and level of expression of Bax protein, this may suggest that oxygen free radicals produced in the cell ,also it can be regulated through the expression of Bax gene to induce cell apoptosis. Bax protein to cause cell mitochondrial cytochrome $\mathrm{C}$ release and Caspase-9, thus inducing the occurrence of apoptosis, however the $\mathrm{Bcl}-2$ protein can inhibit cell mitochondrial cytochrome $\mathrm{C}$ release thus indirectly inhibit Caspase enzyme activity, protect cells so that they don't in the process of apoptosis[21], and can be accessed by the Bcl-2 protein in the cell oxidation mechanism of the oxygen free radical damage caused by cell death. The experimental results show that the exhaustion exercise the expression of Bax proteins in the cerebral cortex of rats, and the Bcl-2 protein expression also increases, but the Bcl-2 protein expression was significantly higher than the increased Bax protein expression increased.

In the motor cortex,the process of cell apoptosis regulation, Bax, Bcl-2 protein and expression of the relationship between BDNF can objectively reflect the body's own protection mechanisms for regulating cell apoptosis. BDNF by regulating the activity of caspase 3 to adjust the Bcl-2, the expression of Bax proteins inhibit motor cortex neuron apoptosis [22].BDNF can adjust promoting apoptosis proteins Bax and apoptosis inhibiting protein expression of Bcl-2 levels, prevent Bax protein to form a homologous dimers and adjust it with the Bcl-2 protein heterologous dimers changes between. The fatigue state of the body under the stimulus of the trauma,motor cortex cell apoptosis increases, in order to maintain normal structure and function of the motor cortex self-protection function plays an important role. After acute exhaustion 
exercise, in the motor cortex of Bax protein expression increased obviously, meanwhile the expression of BDNF also increased, to some extent, increased the expression of BDNF can delay the neuron damage. Increaseing of BDNF promotes the $\mathrm{Bcl}-2$ protein positive cells increased [23].Under this model, the $\mathrm{N}$ group, group $\mathrm{C}$, group D Bax in motor cortex of rats and the Bcl-2 protein expression with the damage increase rise, while the expression of BDNF increased after reaching a certain degree with the motor cortex damage degree aggravating decline, group D BDNF expression decrease may be because of the balance between self protection and damage from the motor cortex stimulation may be broken, motor cortex damage to stimulate more of their self-protection ability.

The results of the experiment show that the myocardial cell and the cortical motor neuron morphology of the rats in fatigue group and sudden death group were abnormal change happened. The myocardial fibers ruptured, vessels dilated abnormally, motor cortex neurons arranged disorder, these pathological changes may be associated with excessive myocardial cell and motor cortex cell apoptosis; Myocardium and brain motor cortex in fatigue state did not recovered fully, increased cell damage, with the phenomenon such as fiber dissolving, abnormal changes in the nerve cell function, there are even myocardial infarction or cerebral infarction. The above changes, could eventually lead to exercise-induced sudden death.

\section{Conclusion}

1. Exercise over fatigue states occurs sudden death then myocardial cell and the cortical motor neuron morphology damaged;

2. Exercise fatigue states occurs sudden death rats myocardial cells Bcl-2,Bax protein abnormal expression,lead to the myocardial tissue damage;at fatigue state occur exercise sudden death,serum LDH-2 levels increased,thus aggravate myocardial injury,leading to myocardial energy dysbolism.

3. The enhanced expression levels of Bax and Bcl-2 proteins and BDNF in brain motor cortex of fatigue and exercise-induced sudden death groups maybe related to the protection-inhibition mechanism. The reduced expression of BDNF in brain motor cortex may be one of the reasons inducing sudden death during over fatigue.

Tip:At the fatigue state, the combined effect of these factors may lead to myocardial cell and nerve cell damage and excessive apoptosis,causing pathological changes in cardiac and brain structure and function,finally induce movement sudden death.

\section{Acknowledgement}

Foundation item:science and technology projects in SiChuan province (experimental reserach of plateau-sub plateau alternative training) Item number: 2014SZ0158

\section{References}

[1] Cheng-JingXue,Zhou-Chun.Sudden death and genetic metabolic disease, Pediatric Clinical Practical magazine[J]2010,25(4):537-539.

[2] Chen-BaoLong, Li-JiLai.A Common cause of sudden death[J].Modern Chinses Medicine Application,2008, 2(8) : 47- 48.

[3] Xu-Xin, Gao-Chong Xuan, Zhang-Shen Li etc. Chinese movement sudden death investigation, Chinese Journal of Sports Medicine [J]1999,18(2):99-102. 
[4] Sun-Qiang.Experimental study of exercise-related cardiac hypertrophy of myocardial ultrastructural changes [J].2013.8(4):33-36.

[5] Yin-Jin,Yue-Jian Xing,Zhou-Jin etc.The research of "excess recovery interval"after glycolysis for exercise energy[J].Journal of ChengDu Sports University,2007,33(4):88-91.

[6-7] Yin-Jin,Jiang-Li,Liu-Qin Long.Exercise Physiology Research Progress of Excess Recovery[M].Electronic Science and Technology Publishing House,ChengDu:2012,4:47-63.

[8] Wever EFD,Rob lesde Med in a EO.Sudden death in patients with out structural heart disease[ J].Am Coll Cardiol, 2004, 43(7) :1137-1144.

[9] Li-Chao, Liu-ShanHong,Zhang-JiaMing, etc. Long-term thyroid hormone stimulation effect on rat myocardial $\mathrm{CaM}$ kinases [J].Chinese Journal Of Pathophysiology, 2012,28(1):147-151.

[10] Zhao-Lin Yang,Wang-Zi Lin,Wu-Jin Yi etc.Exercise related sudden cardiac death clinicopathologic analysis of 9 cases[M].Chinese Journal of Rural Doctor, 1992,2:35.

[11] Wang-Fu Wen,Hu-Zhi Li,Li-Jie.The generation mechanism of exhausted exercise-induced myocardial injury [J].Chinese Journal of Clinical Rehabilitation,2005,28(2):114-116.

[12]Liu-ZhaoQiang,Zhu-JiTian.Mycardial metabolism of lactic acid and acid effects on heart function[J].ChengDu Sports College Journals,1999,(3):57-61.

[13] Wang-Xiao Wei,Cao-Xue Bin,Hou-Cong Cong etc.Clinical study of a theater for training personnel Exhaustive heart damage[J].Chinese Journal of Integrated Traditional and Western Medicine in Intensive and Critical Care,2013,20(5):270-274

[14] Mertens H J,Heinerman M J,Evers J L.The expression of apoptosis-related proteins Bcl-2 and Ki67 in endometrium of ovulatory menstrual cycles[J].Gynecol Obstet,2002,53(4):224-230.

[15] Chen-Xiao Chun, Wen-Zhi Jun,Zhu-Ju Mei.Bcl-2,Bax and Caspase-8 expression in the large amount of myocardial tissue of rats and the Intervention of chitosan oligosaccharide[J].China Sports Science and Technology,2008,44(5):117-120.

[16] Wan-Si Yuan, Cai-Chuan Yuan,Zhang-Yan Qing etc.the effect of levothyroxine and vitamin $\mathrm{E}$ for hypothyroidism myocardial injury[J].Acta Universitatis Medicinalis Anhui.2013,48(1):21-24.

[17] Chi-Su Min, Pei-Jian Min, Liu-Ya Li etc. Endocrine Physiology[M].Fourth Military Medical University Press,XiAn:2006,1:170.

[18] Li-Hao Xia, Wu-Xiao Qiang. Significance of elderly cancer patient serum thyroid hormone testing $[\mathrm{J}]$.ZheJiang Journal Of Integrated Traditional Chinese and Western Medicine,2006,16(7):415.

[19] He-Pei Tian,Gu-Jing Fan,Gao-Lan Xing.The effects of taurine on stress thyroid hormone metabolism of rats [ J] . Journal of Nutrition, 1998, 20(4) : 413- 415 
[20] Zhang-Jun,Xu-Hao Wen,Huang-Shu Huai etc.The effect of Rats Exhaustive Exercise on Mitochondrial Free Calcium and phospholipase A2[J].Chinese Journal of Sports Medicine,1998,17(1):26-27

[21] Mattson M P,Maudsley S,Martin B.BDNF and 5-HT:a dynamic duo in age-related neuronal plasticity and neurodegenerative disorders[J].Trends Neurosci,2004 (27):589-594.

[22] Schabitz WR, Scmmer C, Zoder W, etc. Intravenous brain-derived neurotrophic factor reduces infact size and countenegulates Bax and Bcl-2 expression after temporary focalcerebral ischemia [J].Stroke,2000,31(9):2212-2217.

[23] Sawada M,Nakashimas S,Banno Y,etc.Influence of Bax or Bcl-2 overexpression on the ceramide-dependent apoptotic pathway in glioma cells Oncogene, 2000,19(31):3508. 